\title{
Resumption of Elective Orthopaedic Surgery in the US Epicenter of COVID-19
}

This article was published in the following Dove Press journal:

Orthopedic Research and Reviews

\author{
Eugene S Krauss ${ }^{1-4}$ \\ Ayal Segal ${ }^{1,4}$ \\ Debra Schulman ${ }^{1,4}$ \\ Nancy Dengler' \\ Thomas Bily' \\ MaryAnne Cronin (D) \\ Kathleen Altner ${ }^{4}$ \\ 'Department of Orthopaedic Surgery, \\ Syosset Hospital, Northwell Health, \\ Syosset, NY, USA; ${ }^{2}$ Krauss Musculoskeletal \\ Institute, Peconic Bay Medical Center, \\ Affiliate of Northwell Health, Riverhead, \\ NY, USA; ${ }^{3}$ Zucker School of Medicine, \\ Hofstra/Northwell, Hempstead, NY, USA; \\ ${ }^{4} \mathrm{New}$ York Orthopaedic and Spine Center, \\ Great Neck, NY, USA
}

Correspondence: MaryAnne Cronin Syosset Hospital, Northwell Health, 22I Jericho Turnpike, Syosset, NY I I79I, USA Email mcronin@northwell.edu

\begin{abstract}
On March 1, 2020, New York State confirmed its first case of COVID-19. This state has had the largest initial mortality in the United States with more than 479,000 confirmed cases and over 25,000 deaths as of October 10, 2020. All elective surgeries in New York State were suspended on March 23, 2020, due to the national state of emergency. Syosset Hospital is a 75bed community hospital dedicated primarily to elective surgery. During the COVID-19 surge, the hospital was converted to provide needed beds for the treatment of COVID-19 illness. In anticipation of the resumption of urgent elective procedures, this hospital became one of the two designated sites within the Northwell Health system to be "non-COVID." Once the hospital was emptied of all inpatients, a complete and thorough cleaning and disinfection was performed on the entire building. All equipment was thoroughly decontaminated following Centers for Disease Control and Prevention (CDC) guidelines. In anticipation of the resumption of elective surgery, each surgeon evaluated their cancelled case list to determine patient priority, based on a scale of 1 (elective, non-urgent), 2 (semi-urgent), 3 (urgent), to 4 (highly urgent). Site-specific disaster credentialing was expedited so that emergent surgeries could be performed by surgeons located at other Northwell sites. To ensure a structured and informative onboarding process, each visiting surgeon received a "welcome" email which requested pertinent information to facilitate the surgical process. Presurgical, surgical, and postoperative protocols were revised based on federal and local guidance and regulations. Resumption of elective surgery post COVID-19 placed the hospital into uncharted territory.
\end{abstract}

Keywords: COVID-19, elective surgery, orthopedic surgery, total joint arthroplasty, pandemic

\section{Introduction}

Northwell Health is a nonprofit integrated healthcare network consisting of 23 hospitals and 700 outpatient facilities. With 6600 inpatient beds, it is the ninth largest health system in the United States and the largest in New York State. Based on available data, Northwell Health has treated more COVID-19 patients than any other health system in the nation, caring for over 15,000 hospitalized patients on Long Island, the five boroughs of New York City, and Westchester County, since the outbreak began. ${ }^{1}$ This represents one out of every five COVID-19 patients hospitalized in New York State, nearly double the number of the next-highest health system. When Governor Andrew Cuomo ordered hospitals to create more beds, Northwell Health hospitals added nearly 2000 additional beds within the span of about 10 days.

On March 1, 2020, New York State confirmed its first case of COVID-19. The state had the largest initial outbreak in the United States with more than 474,000 confirmed cases and 25,000 deaths as of October 10, 2020., ${ }^{2,3}$ On March $11^{\text {th }}$, the 
World Health Organization (WHO) categorized the COVID19 outbreak as a pandemic, and two days later, the President of the United States declared a national state of emergency, including a recommendation to curtail elective procedures. ${ }^{4}$ The American College of Surgeons provided a similar endorsement on March $13^{\text {th. }}$ New York stepped beyond the federal recommendation, and placed a moratorium on elective surgery due to the crisis facing the state. ${ }^{6}$ All elective surgeries in New York state were suspended on March 23, 2020. ${ }^{7,8}$ During the COVID-19 surge, the hospital was converted to provide needed beds for the treatment of COVID-19 illness (Figure 1).

\section{Background}

Syosset Hospital is a 75-bed community hospital dedicated primarily to elective surgery, including orthopaedic, bariatric, and ophthalmology specialties. More than 1000 total joint arthroplasties are performed annually. Overall, elective procedures account for about $50 \%$ of hospital system costs, and potentially an even higher return of hospital revenue. ${ }^{9}$ The loss of three months of elective surgery has been estimated to decrease annual hospital revenue by $12.5 \%{ }^{10}$ In addition to the financial losses, it was predicted that the need for surgical care would be massive once the New York metropolitan area had moved past the first wave of the pandemic, and it was recommended that hospitals ready their facilities to meet this demand. ${ }^{11}$ Thus, shortly after discontinuation of elective surgery, planning was initiated to safely and efficiently resume elective orthopaedic surgery when approved by federal and local authorities. Facing a backlog of over 5500 patients in need of orthopaedic surgery after two months' time, Northwell Health began to look ahead in preparation for the reinitiation of elective surgery once appropriate criteria were met. ${ }^{12}$

Syosset Hospital was one of two sites designated within Northwell Health to be "non-COVID" to provide a safe environment to resume urgent elective procedures. On April 22, 2020, Northwell Health executed a wellorchestrated, system-wide collaboration to methodically vacate Syosset Hospital of all patients. Stable patients were discharged to home or to subacute rehabilitation facilities, and all remaining inpatients were transferred to other health system locations, where beds were available. The Emergency Department (ED) remained open to accept patients. Patients were transferred to other Northwell Health sites if they required hospital admission. Personal Protective Equipment (PPE) including the use of N-95 masks, gowns, goggles/face shields, and gloves are worn in the ED at all times.

\section{Pre-Opening/Cleaning/Sterilization Process}

The multifactorial, multidisciplinary process of resuming elective surgery begins here (Figure 2). Once the hospital was emptied, a complete and thorough cleaning and disinfection was performed on the entire building. All equipment was thoroughly decontaminated following Centers for Disease Control and Prevention (CDC) guidelines. ${ }^{13,14}$

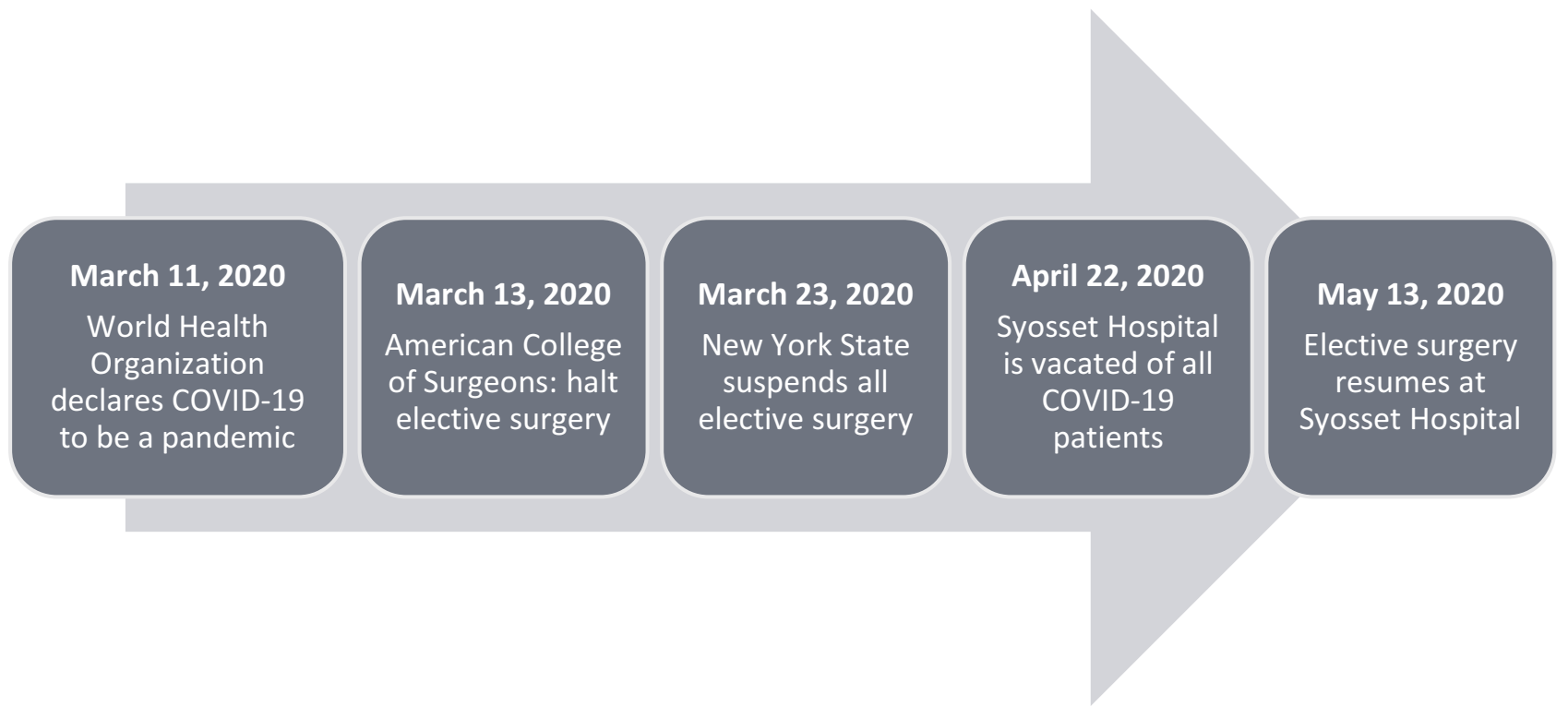

Figure I Timeline of events in New York State as epicenter of COVID-19 in the US. 

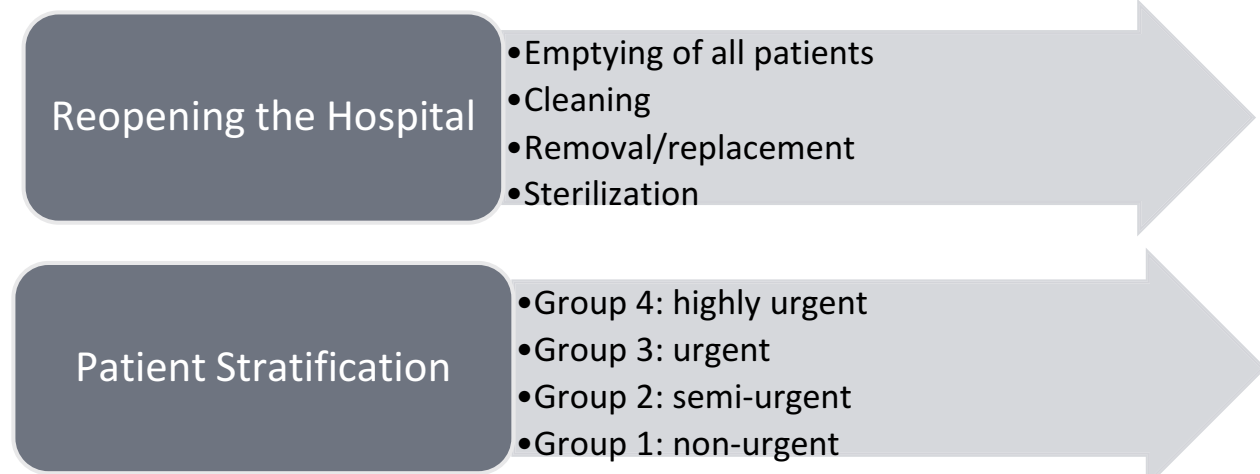

- Group 4: highly urgent

- Group 3: urgent

- Group 2: semi-urgent

- Group 1: non-urgent

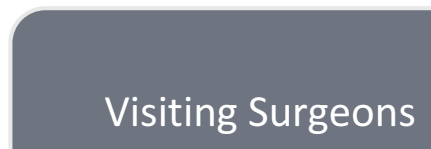

- Disaster credentialing

- Communication of operating room needs

- Team provided with specific protocols

- Onsite orientation prior to first case

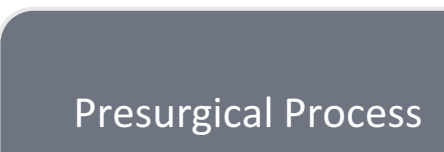

- Online preoperative education

- History/Medical clearances

-PCR testing within 48 hours of surgery

-Admission physical morning of surgery

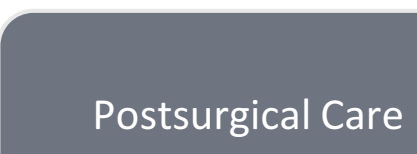

- Twice daily team rounds

- Multidisciplinary postsurgical management

- Necessary education provided to family

- Discharge home

Figure 2 Process to reopen a "non-COVID" hospital after COVID-19 surge for elective surgery.

Curtains were removed from patient care areas and replaced with new ones. All areas (ie, hallways, patient rooms, offices, operating rooms (ORs), and waiting areas) of the hospital were terminally cleaned with Environmental Protection Agency (EPA)-approved chemicals by our environmental service (EVS) team members. An electrostatic machine was loaned to Syosset Hospital by the Clorox Company (Oakland, California) and was used in all patient rooms, ORs, and on medical equipment. This electrostatic technology entails an electrically charged chemical that forces the product to adhere to all surfaces, eliminating human error. Degmor Environmental Services (Brooklyn, New York) was brought in for additional support. They cleaned all hospital surfaces and utilized fogging technology in all areas of the hospital. Ultraviolet (UV) technology was also brought in, through the generosity of Xenex Disinfection Services (San Antonio, Texas), who loaned Syosset Hospital a robot for the cleaning process. After UV technology was utilized, all areas of the hospital were terminally cleaned again by EVS team members. Hallways and patient room floors were stripped and re-waxed. Carpeting was replaced throughout the building. The entire cleaning process spanned a five-day period.

\section{Patient Stratification and Prioritization}

Elective surgery resumed on May 13, 2020. In anticipation of this go-live date, each surgeon evaluated their cancelled case list to determine patient priority, based on a scale of 1 (elective, non-urgent), 2 (semi-urgent), 3 (urgent), to 4 (highly urgent). During the downtime, surgeons kept in contact with their patients, assuring them that planning was ongoing for the restart of elective surgery. Metrics used to identify the first patients to be scheduled were based on these criteria:

- Review of X-ray for severity of disease: imminent failure of joint, osteonecrosis, or risk for severe deformity 
- Body Mass Index $<40$

- Uncomplicated medical history (eg, controlled diabetes, absent/stable cardiac disease)

- Inability to perform activities of daily living (ADLs)

- Visual Analog Scale (VAS) pain score 8 to 10 (out of 10)

- COVID-19 negative and no known symptoms

- No family member meeting screening criteria for COVID-19 testing

- Ability/willingness to complete an online preoperative education class

- No chronic medications that required interruption (eg, anticoagulants, immunosuppressants)

- Support system in place for a safe discharge home on post-operative day (POD) 1 or 2

Patients were scored as a Level 4 (highest) if their pain level was between 8 to 10 (out of 10), they were immobile or were house bound, and their x-ray showed severe disease. These patients were contacted first to schedule surgery. The authors found that some patients declined to move forward with their surgery due to concerns about hospitalization during the ongoing pandemic even though this institution was "non-COVID". Many of the concerns were not only due to the hospital stay, but related to the preoperative and postoperative processes. Patients often did not feel comfortable going for needed medical clearances. Additionally, family members were often not available to assist with the postoperative care. Some patients were apprehensive to allow nursing services and physical therapy into their home following discharge. "Sicker" patients not meeting the original criteria have been cleared for surgery as post-COVID processes have become smoother.

\section{Preparation Process for Visiting Surgeons}

Site-specific disaster credentialing was expedited so that emergent surgeries could be performed by surgeons located at other Northwell sites. Although "visiting" surgeons were new to Syosset Hospital, they were already credentialed by other Northwell Health system facilities. To ensure a structured and informative onboarding process, each visiting surgeon received a "welcome" email, which requested pertinent information to facilitate the surgical process:

- Surgeon contact information
- Photo of visiting surgeon and assistants to expedite building and scrub access

- Scrub size

- Need for first assistant in the OR

- OR "preference cards" to assist nurses with the setup of instruments and supplies

- Communication of specific preoperative and postoperative clinical protocols

As with all new initiatives, communication is paramount for effective and safe care. Sharing the surgeon's contact information with hospital leadership and clinical teams was pivotal. The visiting surgeons were also provided with a comprehensive contact list for the key site leaders.

The visiting surgeon and members of their teams emailed a photograph of their Northwell ID badge along with their scrub size to a point person. This email was then forwarded to site Human Resources as well as the Material Management supervisor to provide access to the building and scrub machines.

All surgeons were asked if they would need a first assistant in the OR. A total of fifty-seven percent of all visiting surgeons provisioned their own surgical assistant and the other forty-three percent utilized incumbent surgical Physician Assistant (PA) staff. Surgeons were placed in contact with our Surgical Program Manager to provide their intraoperative preferences.

Providing an orientation to our facility was an important facet to the success of this initiative. A brief orientation communication was emailed to each visiting surgeon and surgical assistant. Additional on-site orientations occurred on the day of the first scheduled case or in the days prior upon request. Email orientation helped communicate the following:

- Parking accommodations

- Entrance information including temperature scanning

- Process of obtaining N-95 masks

- Medical-surgical co-management model

- Instructions to the physician dictation system

- Information about our "Meds to Beds" pharmacy program

In order to provide an easier workflow process, our on-site dictation system was made available to the visiting surgeons. Intra-facility dictation systems vary throughout the Northwell Health system. Our Director of Health Information Management provided a dictation system 
"cheat sheet," which was attached to the welcome email. Direct contact information for assistance with the dictation system was also provided to each surgeon.

\section{Presurgical Process (Education, Evaluation, Admission)}

The preoperative process required revision as well. All patients undergoing joint replacement surgery are required to complete a preoperative education program. Since the classroom version has been suspended due to COVID-19, patients scheduled for surgery are required to complete a mandatory online program. A link is now available on the hospital's orthopaedic department webpage, and visiting surgeons have the option to use this video for their own patients. Prior to their surgery date, patients are called by the nurse educator to confirm that they had viewed the video, and are asked a few simple questions regarding the content of the program. Six months after resumption of elective surgery, this process remains active, with no inperson education offered to patients. Based on patient feedback, a COVID-19 specific video has been created to review additional pandemic precautions currently in place.

Pre-Surgical Testing (PST) visits have been suspended. A comprehensive medical history is obtained by the PST nurse practitioner via telephone interview with the patient and/or family member. A COVID-19 nasal swab is performed 48 hours prior to the day of surgery, and the surgery is cancelled if the polymerase chain reaction (PCR) result is positive. Patients are told to quarantine at home after PCR swab is performed until surgery. The physical portion of the evaluation is then completed upon patient arrival to the preoperative unit, prior to any other procedures. This has added mandatory steps to the busy preoperative time period but with no additional time added. The preoperative nurses are consummate professionals and have embraced the necessity of this new procedure, despite the added strain on their responsibilities within the same time constraints. Lab work, EKGs, and a chest x-ray are performed as needed. Patients are given the option of having a medical clearance completed by their primary care provider (PCP) within 30 days of surgery, or by a staff hospitalist on the day of surgery. This is necessary as some PCP offices have been slow to open their offices for pre-surgical visits, or in some cases, patients have been uncomfortable going into a medical office with the risk of possible COVID-19 exposure.
Admission documents are completed remotely via telephone. Required signatures are obtained on the day of surgery in the patient waiting room. Patients are only escorted into the Admitting Office if a collection of payment is required. All patient chairs have been removed to limit the time spent in the Admitting Office, and no more than two patients at a time are allowed in order to maintain social distancing.

Limiting traffic within the hospital remains a top priority. To maintain our "non-COVID" status, visitation is suspended. Staff are located at all hospital entry doors to direct patients to the main entrance and assist as needed. Patients scheduled for surgery may only enter the hospital through the main lobby, and not through the Emergency Room entrance. Staff and patients have temperature checks with a remote scanner when entering the building. Any patient or staff member with a temperature above $100^{\circ} \mathrm{F}$ is denied access. Surgical masks are required for all staff and patients. These are provided at the front desk. Patients are also instructed to clean their hands with hand sanitizer when entering the hospital.

\section{Postsurgical Patient Care}

Our orthopaedic service includes a dedicated team of surgeons, anesthesiologists, certified nurse anesthetists, physician assistants, nurse practitioners, surgical nurses, hospitalists for medical co-management, floor nurses, patient care associates, as well as physical and occupational therapists. Our program is comprehensive in nature and includes a dedicated clinical pharmacist, research nurse and wellness coordinator. In addition, our orthopaedic department follows one standardized, evidence-based protocol, including perioperative antimicrobial prophylaxis, prevention and treatment of postoperative nausea and vomiting (PONV), multimodal pain management, blood management (ie, intraoperative tranexamic acid), and risk stratification using the Caprini Risk Assessment Model (RAM) ${ }^{15,16}$ for thromboprophylaxis. A detailed Transition of Care education video and booklet are provided to each patient, along with a patient-specific medication calendar to assist with medication compliance after discharge.

All visiting surgeons are offered the option to follow Syosset Orthopaedic Department pharmacologic protocols, which is shared with them in advance of their first scheduled surgery. A discussion between the clinical pharmacist and visiting surgeon assists in smoothing the process. A surgeon-specific worksheet has been created to highlight protocol similarities and differences for professional staff. 
These worksheets have been reviewed by primary stakeholders and are then distributed to key areas for access by the entire team, providing detailed information to minimize phone calls and confusion.

Our clinical care model consists of a 24/7 in-house coverage by a hospitalist team, as well as general surgery, orthopaedic surgery, and critical care physician assistant services. Surgical rounds have been increased to twice a day (morning plus afternoon debrief) to ensure that communication is maintained among team members. This is particularly important right now, as we have several visiting surgeons in the building with protocols unfamiliar to our staff. Postoperatively, all patients are admitted to private rooms. Length of stay is anticipated to be one day, unless there is a medical or surgical reason to delay discharge. Length of stay since return after the COVID-19 surge is 1.5 days, reduced from 2.5 days prior to COVID-19.

COVID-19 is a disease of inflammation with associated hypercoagulability. In addition to the requirement to be COVID-19 negative prior to surgery, patients are screened for a history COVID-19 positivity and/or symptoms highly suspicious of COVID-19. This is done to assess patient exposure to the disease and to identify the potential for postoperative complications related to increased thrombotic risk, as we still do not know how long body systems are altered by this virus. Dr. Joseph Caprini, an international expert on thromboembolism and risk stratification, recommends to account for the increased risk of thrombosis associated with the COVID-19 virus. ${ }^{17}$ This is accomplished by adding 2 additional points to the Caprini Risk Assessment Model in patients who have had COVID-19 infection within the last 6 months. To date, we have had no patients develop a venous thromboembolism (VTE), and all patients are called at 60 days postoperatively to capture outcomes.

Discharge to a rehabilitation center is highly discouraged due to concerns of COVID-19 outbreaks in these facilities. Patients have been made aware preoperatively that they will be discharged to home on POD 1. Patients are required to designate a caregiver prior to surgery. We have found, although rarely, that postoperatively some patients cannot be discharged safely to the home environment, requiring subacute rehabilitation. After finally achieving control of the COVID-19 surge in New York combined with strict regulation of these facilities to suppress internal viral spread, many of the subacute facilities are now non-COVID or have separate non-COVID areas. Over the 6 months since resumption of surgery, $3.8 \%$ of patients have required subacute rehabilitation. This is in stark comparison to $14.5 \%$ prior to post-pandemic resumption.

The workload for hospital case managers has increased markedly, since most patients are going directly home after surgery. This has required the reallocation of resources to address the added responsibility of these team members. Durable Medical Equipment (DME) such as rolling walkers, commodes, and hip kits are now ordered and delivered to the patient's home prior to hospitalization. Providing home care services to this patient population is not an issue since they are all confirmed to be COVID-19 negative prior to hospital admission. Virtual rehabilitative services are available to any patient who is uncomfortable with in-home physical therapy or multiple visits to an outpatient rehabilitative center. Patients who choose to engage in virtual rehabilitation receive one initial evaluation by a physical therapist to develop a patient-specific treatment plan. They are then able to continue with their home exercise program using this remote technology, which is closely monitored by their physical therapist. Communication between the physical therapist and the surgeon is paramount in order to monitor the patient's progression and recovery process.

Incorporating relevant caregivers into the educational and discharge processes is vital to a successful surgical outcome. This is a new challenge as no visitors are allowed in the hospital. This is accomplished by utilizing either Facetime or telephone discussion with the discharge team, based on patient preference. In addition, Syosset Hospital offers a pharmaceutical service called "Meds-toBeds," whereby postoperative prescriptions are sent to a centralized retail pharmacy and delivered to the patient's bedside prior to discharge home. This eliminates the need for the patient to visit their local pharmacy. At discharge, patients are escorted outside the building and picked up by a family member.

On May $13^{\text {th }}$, the first day elective surgery resumed, procedures were limited to only 4 , to test the newly implemented processes. For the remainder of May 2020, 38 surgeries were performed. Since then, the number of monthly procedures has ranged from 111 to 125 (June through October 2020).

\section{Conclusion}

Resumption of elective surgery post COVID-19 has placed us into uncharted territory. Processes that existed prior to COVID-19 are no longer applicable in this new environment and we continue to utilize these "post-COVID" practices described above. We continue to navigate through the 
surgical setting, responding to guidance from local and federal authorities while monitoring emerging literature to provide safe and effective perioperative care. As the world learns more about this new disease called COVID-19, we anticipate ongoing revisions to our processes. Healthcare has never been more challenging. Whether facing a personal or societal crisis, one should always wonder if it is possible to emerge from the event in a better place than before. The message is to redirect resources, listen to and learn from each other, reassure patients that their health and well-being are our top priority, expand our compassion for those in need, and allow these uncertain times to transform us into finer healthcare providers. With good leadership, some foresight and a dedicated team of professionals, we will ensure optimal patient outcomes as we resume elective orthopaedic surgery.

\section{Acknowledgments}

Stephen Bello, Michael Fener, John Bodolai, Verona Henningham, John Schiliro, Regina Bracco, Maria Corpuz, Nancy Gawrych, Gary Isaksen, Carol Lampros, Stacy Lanza, Jeanine Norton, Lisa Schiraldi.

\section{Funding}

The study was self-funded by the Department of Orthopaedic Surgery at Syosset Hospital.

\section{Disclosure}

The authors report no conflicts of interest for this work.

\section{References}

1. Northwell Health Analytics, Krasnoff Institute. COVID-19 Data.

2. New York State Department of Health COVID-19 tracker. Available from: https://covid19tracker.health.ny.gov/views/NYS-COVID19Tracker/NYSDOHCOVID-19Tracker-Map?\%3 Aembed=yes\&\% 3Atoolbar $=$ no\&\%3Atabs $=n$. Accessed June 12, 2020.

3. New York State Department of Health COVID-19 fatalities. Available from: https://covid19tracker.health.ny.gov/views/NYS-COVID19Tracker/NYSDOHCOVID-19Tracker-Fatalities?\%3Aembed=yes\&\% 3 Atoolbar $=$ no\&\%3Atabs $=$ n. Accessed June 12, 2020.
4. Trump DJ Proclamation on declaring a national emergency concerning the novel coronavirus disease (COVID-19) outbreak. 2020. Available from: https:/www.whitehouse.gov/presidential-actions/pro clamation-declaring-national-emergency-concerning-novelcoronavirus-disease-covid-19-outbreak/. Accessed April 7, 2020.

5. American College of Surgeons. COVID-19: recommendations for management of elective surgical procedures. March 13, 2020. Available from: https://www.facs.org/covid-19/clinical-guidance/elec tive-surgery. Accessed December 11, 2020.

6. Setting PIS, Phase P. COVID-19: guidance for triage of non-emergent surgical procedures. American College of Surgeons. 2020 Mar 17. Available from: https://www.facs.org/about-acs/covid19/information-for-surgeons/triage. Accessed April 7, 2020.

7. Executive Order No. 202.10, Governor Andrew Cuomo, Continuing temporary suspension and modification of laws relating to the disaster emergency. Available from: https://www.governor.ny.gov/sites/gover nor.ny.gov/files/atoms/files/EO_202.10.pdf. Accessed December 11, 2020.

8. New York State Department of Health. COVID-19 directive regarding the resumption of elective outpatient surgeries and procedures in general hospitals in counties and facilities without a significant risk of COVID-19 surge. Available from: https://coronavirus.health.ny.gov/ system/files/documents/2020/05/elective-surgerynotification_4_29_20.pdf. Accessed December 11, 2020.

9. Nicholson S, Ash DA Hospitals need cash. Health insurers have it. 2020 Mar 25. Available from: https://hbr.org/2020/03/hospitals-needcash-health-insurers-have-it.Accessed April 7, 2020.

10. Center for Medicare \& Medicaid Services. National health expenditure data. 2019. Available from: https://www.cms.gov/ResearchStatistics-Data-and-Systems/Statistics-Trends-and-Reports /NationalHealthExpendData/NationalHealthAccountsHistorical. Accessed March 42020.

11. Joint Statement: Roadmap for Resuming Elective Surgery After COVID-19 Pandemic. American College of Surgeons, American Society of Anesthesiologists, Association of perioperative Registered Nurses, American Hospital Association.

12. American College of Surgeons: Guidance for resumption of elective surgery. Local Resumption of Elective Surgery Guidance. April 17, 2020 Available from: https://www.facs.org/-/media/files/covid19/ local_resumption_of_elective_surgery_guidance.ashx. Accessed December 11, 2020.

13. https://www.cdc.gov/coronavirus/2019-ncov/community/cleaningdisinfecting-decision-tool.html. Accessed December 11, 2020.

14. https://www.cdc.gov/coronavirus/2019-ncov/community/organiza tions/cleaning-disinfection.html. Accessed December 11, 2020.

15. Illinois Medical Society. https://www.venousdisease.com/caprini-dvtrisk-assessment.pdf. Accessed December 11, 2020.

16. Krauss ES, Segal A, Cronin M, et al. Implementation and validation of the 2013 caprini score for risk stratification of arthroplasty patients in the prevention of venous thrombosis. Clin Appl Thromb Hemost. 2019;25:1076029619838066. doi:10.1177/1076029619838066

17. Personal communication, JA Caprini.
Orthopedic Research and Reviews

\section{Publish your work in this journal}

Orthopedic Research and Reviews is an international, peer-reviewed, open access journal that focusing on the patho-physiology of the musculoskeletal system, trauma, surgery and other corrective interventions to restore mobility and function. Advances in new technologies, materials, techniques and pharmacological agents are particularly welcome. The manuscript management system is completely online and includes a very quick and fair peer-review system, which is all easy to use. Visit http://www.dovepress.com/testimonials.php to read real quotes from published authors. 